\title{
Association between dietary patterns and low bone mineral density among adults aged 50 years and above: findings from the North West Adelaide Health Study (NWAHS)
}

\author{
Yohannes Adama Melaku ${ }^{1,2 *}$, Tiffany K. Gill ${ }^{1}$, Robert Adams ${ }^{3}$ and Zumin $\mathrm{Shi}^{1}$ \\ ${ }^{1}$ Population Research and Outcome Studies, School of Medicine, The University of Adelaide, SAHMRI, Adelaide, \\ SA 5005, Australia \\ ${ }^{2}$ School of Public Health, Mekelle University, Mekelle, Ethiopia \\ ${ }^{3}$ Health Observatory, Discipline of Medicine, The Queen Elizabeth Hospital Campus, The University of Adelaide, Adelaide, \\ SA 5011, Australia
}

(Submitted 10 June 2016 - Final revision received 7 August 2016 - Accepted 21 August 2016 - First published online 27 September 2016)

\section{Abstract}

Studies on the association between dietary patterns and bone mineral density (BMD) have reported inconsistent findings. Data from the North West Adelaide Health Study, a population-based cohort study undertaken in Australia, were used to assess this association among adults aged 50 years and above. In this specific study, 1182 adults (545 males, 45.9\%) had dietary data collected using a FFQ and also had BMD measurements taken using dual-energy X-ray absorptiometry. Factor analysis with principal component method was applied to ascertain dietary patterns. Two distinct dietary patterns were identified. Pattern 1 ('prudent pattern') was characterised by high intake of fruits, vegetables, sugar, nut-based milk, fish, legumes and high-fibre bread. In contrast, pattern 2 ('Western pattern') was characterised by high levels of processed and red meat, snacks, takeaway foods, jam, beer, soft drinks, white bread, poultry, potato with fat, high-fat dairy products and eggs. Compared with the study participants in the first tertile (T1, lowest consumption) of the prudent pattern, participants in the third tertile (T3) had a lower prevalence of low BMD (prevalence ratio (PR) $=0.52 ; 95 \%$ CI 0.33, 0.83) after adjusting for socio-demographic, lifestyle and behavioural characteristics, chronic conditions and energy intake. Participants in T3 of the Western pattern had a higher prevalence of low BMD ( $\mathrm{PR}=1.68 ; 95 \% \mathrm{CI} 1.02,2.77$ ) compared with those in $\mathrm{T} 1$. In contrast to the Western diet, a dietary pattern characterised by high intake of fruits, vegetables and dairy products is positively associated with BMD.

Key words: Dietary patterns: Bone mineral density: Adults: Australia

Bone is a dynamic tissue comprised of cellular, organic and inorganic components with a complex internal structure. Disruption of the balance between bone formation and resorption due to excessive production of osteoclasts or inadequate presence of osteoblasts leads to bone loss, and hence osteoporosis ${ }^{(1)}$. The level of osteoporosis is increasing worldwide, with more than 200 million people living with osteoporosis in $2010^{(2)}$. Despite underestimations in reports of osteoporosis prevalence ${ }^{(3)}$, available epidemiological evidence has shown that the magnitude has increased in Australia. In $2012,3.3 \%(5.3 \%$ men and $1.2 \%$ women $)$ of the general Australian population self-reported that they had osteoporosis, which was double the estimate of $1.6 \%$ from the year 2000 . This figure was higher among those aged 50 years and above (15 and $3 \%$ in women and men, respectively) $)^{(4)}$.

Determinants of osteoporosis are multifaceted and interlinked. Genetic, lifestyle, nutritional, medical disorders, medication use and metabolic (biological) risks are identified as major contributors for osteoporosis ${ }^{(5)}$. Evidence has demonstrated the importance of specific food items, nutrients and nonnutritive substances in maintaining bone mineral density (BMD) and preventing osteoporosis and osteoporotic fractures ${ }^{(6)}$. For instance, high consumption of soya ${ }^{(7)}$ and dairy products ${ }^{(8)}$ has been found to be important in the prevention of osteoporosis. Nutrients and non-nutritive substances, such as $\mathrm{Ca}^{(9)}, \mathrm{PUFA}^{(10)}$ and isoflavones ${ }^{(11)}$, were also found to have an important role in the prevention of osteoporosis.

Recent epidemiological studies have focused on the effect of the overall nature of food consumption habits on disease outcomes, instead of specific foods or nutrients ${ }^{(12)}$. This is because an outcome (disease) usually occurs as a result of natural interactions or patterns of nutrients and other components of diets rather than intake of single foods or nutrients. In line with this, new dietary analysis methods have been introduced. These

Abbreviations: BMD, bone mineral density; DXA, dual-energy X-ray absorptiometry; EI, energy intake; PAL, physical activity level; PR, prevalence ratio; NWAHS, North West Adelaide Health Study.

* Corresponding author: Y. A. Melaku, fax +61 88313 1218, email yohannes.melaku@adelaide.edu.au 
methods are either 'a posteriori' analyses (data driven techniques), such as factor, principal component and cluster analysis, or 'a priori' analyses, which include dietary indices or dietary scores. Most recently, reduced-rank regression, which combines the above two, has also been used ${ }^{(13)}$.

Studies have reported different patterns of diet that are associated with BMD ${ }^{(14,15)}$. However, findings are inconsistent. For example, a study in South Korea among postmenopausal women showed a direct association between dairy-rich dietary patterns and $\mathrm{BMD}^{(14)}$. However, another study found no such association among Canadian women ${ }^{(15)}$. The effect of dietary patterns on BMD can also vary by communities as the food that is available in one location may not be found in others. Hence, tailored dietary patterns that are useful for optimal bone mass should be developed for specific groups/populations.

In Australia, a few studies have explored the association between dietary patterns and BMD, and the available studies are generally conducted among children and young adults $^{(16,17)}$. The present study, therefore, aims to assess the association between dietary patterns and low BMD among adults aged 50 years and above in Australia.

\section{Methods}

\section{Study design and population}

The North West Adelaide Health Study (NWAHS) data were used for this analysis. The NWAHS recruited participants from the northern and western suburbs of Adelaide, South Australia. The region represents a third of the South Australian population and half of the metropolitan area of the city of Adelaide, and was established with the purpose of providing valid and reliable data on chronic diseases and their risk factors. It is a community-based cohort study that incorporates clinical, public health, social and biochemical data. Three stages of data collection have been conducted: 1999-2003, 2004-2006 and 2008-2010. Data were collected a using self-completed questionnaire, computer-assisted telephone interview and clinical assessments ${ }^{(18)}$.

Details on the objectives and methods of the NWAHS are published elsewhere ${ }^{(18)}$; however, in brief, the study participants were adults aged 18 years and above when first recruited. Random sampling was initially undertaken at the household level. All households that were not connected to a landline telephone were excluded from the sampling frame (using Electronic White Pages). At the time of recruitment (1999), 97.9\% of households in South Australia were connected with a landline telephone ${ }^{(19)}$. Randomly selected households were screened for individuals aged 18 years and above. All these individuals were then invited to participate in the study. Those who could not communicate in English were excluded. At the initial stage, 4056 males and females participated. This study used BMD data collected from those aged 50 years and over as part of Stage 2 (2004-2006, $n$ 1588), and dietary data was collected as part of Stage 3 (2008-2010, $n$ 2500). In total, 1182 adults ( 545 males, $45.9 \%$ ) aged 50 years and above provided data related to BMD and nutrition.

\section{Dietary assessment and food groups}

Dietary intake was assessed using the Dietary Questionnaire for Epidemiological Studies (DQESV2) of Cancer Council of Victoria. The DQESV2 was self-completed and designed to assess intake over the preceding 12 months. Portion size was assessed using four questions and by calculating a single portion size factor, which helps in estimating a median-sized serving of food an individual eats ${ }^{(20)}$. The completed forms were sent to Cancer Council Victoria for analyses of total daily intakes of food items and nutrients using the Australian NUTTAB95 (Australian Government Publishing Service, Canberra) food composition database. The amount of food items consumed per day was calculated in grams for each study participant. Food items were categorised into thirty-nine food groups ${ }^{(21)}$. Data on vitamin D and Ca supplementation were also collected.

\section{Assessment of other covariates}

Stage 2 covariates. Sex, age and family history of osteoporosis were determined. Annual household income was categorised as follows: up to $\$ 20000, \$ 20001-\$ 40000, \$ 40001-\$ 60000$ and more than $\$ 60000$. Marital status was determined and categorised into married or living together with partner (in union), separated/divorced, widowed and never married. Alcohol intake risk was assessed using the frequency and number of standard drinks ${ }^{(22)}$. Smoking status was classified into nonsmokers, ex-smokers and current smokers. Height and weight of the study participants were obtained to calculate the BMI. BMI was further classified on the basis of the World Health Organization standard ${ }^{(23)}$. Identification of participants with diabetes was by either doctor-diagnosed self-report of diabetes or laboratory diagnosis using blood samples collected during the clinic visit, with diabetes defined as fasting plasma glucose $\geq 7 \cdot 0 \mathrm{mmol} / 1$.

Assessment of leisure-time physical activity levels (PAL) was performed using the Australian National Health Survey questions ${ }^{(24)}$. This was assessed considering the number of times a person exercised in the last 2 weeks and the total amount of time spent walking for exercise and performing moderate and vigorous exercise. Job-related PAL was also assessed from data related to occupation, which were obtained in Stage 1, by two occupational physicians based on the type of professions the study participants had. Both PAL were classified as sedentary or low and medium or high. In this particular analysis, home duties were considered as sedentary or low PAL. Detailed methods of both PAL are published elsewhere ${ }^{(25)}$. Total number of medications prescribed over the past 6 months (including for hypertension, high cholesterol, mental health problems, osteoporosis and asthma), menopausal status and sunlight exposure were also assessed at this stage. Data on medication use were obtained from pharmaceutical benefits scheme. Sunlight exposure was assessed using questions including average duration of direct sunlight exposure during winter and summer and timing (week day and weekend).

Stage 3 covariates. Health literacy was assessed using the Newest Vital Sign test tool ${ }^{(26)}$. For thirty-one cases with missing 
values, we used data collected with the short Test of Functional Health Literacy in Adults tool ${ }^{(27)}$. Health literacy was classified as limited or adequate.

\section{Assessment of bone mineral density}

BMD of the whole body was measured using Prodigy and DPX + dual energy X-ray absorptiometry (DXA) (GE Lunar) as part of the clinic visit at stage two. DXA was calibrated, and measurements were verified to check correct operation at the beginning of each scan day. Details of the DXA measurement procedures can be found elsewhere ${ }^{(28)}$. Participants were categorised into two groups using $T$-scores of BMD. Those who had T-scores of less than -1 were considered as osteopenic (between -1 and $-2 \cdot 5$ ) or osteoporotic (less than or equal to $-2 \cdot 5)^{(29)}$ and were classified as having low BMD.

\section{Dietary and statistical analysis}

To evaluate dietary misreporting, the Goldberg method was used. In this method, the ratio of actual energy intake (EI):BMR and PAL were considered ${ }^{(30)}$. To take account of variations in methods, the $95 \%$ CI of PAL was calculated. Both leisure-time and job-related PAL were considered in the calculation. Next, the ratio of EI:BMR was compared against the $95 \% \mathrm{CI}$ of PAL. On the basis of the recommendation by Black et al., the following values for PAL were determined: sedentary $=1.4$, light $=1.6$, medium $=1.8$ and strenuous $=2$. Individuals were classified as plausible if the ratio was in the CI range. However, if the ratio was below or above the $95 \% \mathrm{CI}$, it was classified as under-reported or over-reported, respectively ${ }^{(31)}$.

To represent population-level dietary patterns, factor scores and dietary patterns were calculated and constructed among 2453 (forty-seven cases with considerable (>30) missing values were excluded) study participants who provided dietary information. Data reduction technique using factor analysis with principal component analysis was used to identify dietary patterns out of the thirty-nine food groups; two dietary patterns were determined on the basis of the scree plot, eigenvalues $(>1)$ and interpretability. To attain optimal structure and increase the interpretability of factors, varimax rotation was applied. Factor scores for each of the participants and factors were calculated as the sum of the products of factor loading coefficients, which was standardised by daily intake of each food item. Tertiles were constructed for each factor. Factor loadings are the correlation coefficients between factors (identified dietary patterns) and food groups with loadings of each food group graphically presented. Sample adequacy was checked using the Kaiser-Mayer-Olkin test.

Descriptive analysis of socio-demographic and lifestyle characteristics and chronic conditions was performed across the tertiles of the factors. Mean values and standard deviations (continuous and normally distributed variables), medians and interquartile ranges (continuous and non-normally distributed variables) and proportions were calculated (categorical variables). $\chi^{2}$ Tests, Kruskal-Wallis tests and ANOVA were used to identify significant differences across different levels of dietary intake (factor scores).
To assess the association between intake of different levels of dietary patterns and low BMD, Poisson regression models were used ${ }^{(32)}$. For both dietary patterns, we developed four regression models in addition to the unadjusted model. The first model was adjusted for sex and age. Model two was additionally adjusted for socio-economic and lifestyle factors (smoking status, alcohol intake, marital status, income, health literacy and job-related PAL). In addition to the variables in the second model, chronic conditions (diabetes mellitus, family history of osteoporosis and BMI) were adjusted in the third model. To assess whether the association between dietary patterns and outcomes was confounded by total EI, we additionally adjusted for EI in the fourth model.

Subgroup analyses were performed to assess the association of dietary patterns with low BMD in various subgroups of the study participants. In the final models, multiplicative terms for each dietary pattern and each of the variables were used to assess the interaction in predicting low BMD. Missing data were identified across all variables. Except for leisure-time PAL, which had the highest number of missing values, others were imputed using data from the other stage of the study or were otherwise reported as 'missing'. We did not impute leisure-time PAL in the analysis because the approaches used to assess the PAL at different stages were not the same, and it had a high number of missing values ( $n$ 128). A sensitivity analysis was undertaken by including and excluding the missing values and variables, including season of birth and DXA measurement, leisure-time PAL, vitamin D and Ca supplementation, menopausal status, medication use and sunlight exposure, in the final models. All analyses were conducted using STATA/SE version 14.1 (Stata, StataCorp LP).

\section{Results}

A total of 1182 (45.9\%, males) study participants provided dietary and BMD data and were included in the analysis. However, the total number of study participants in the multivariable analysis was 1066. Therefore, 119 (9.8\%) cases had at least one missing value among the other covariates. Variables such as leisure time PAL (128, 10.8\%) and health literacy (34, $2.9 \%$ ) had the highest proportion of missing values (Table 1). Missing values of variables including smoking status (five cases), alcohol intake risk (thirty-nine cases), diabetes (five cases), family history of osteoporosis (four cases) and marital status (four cases) were identified and imputed using data from the third stage.

\section{Socio-demographic characteristics}

The median age of the participants at the second stage of assessment was 62 years (interquartile range 56.0, 69.0). Almost half $(47.7 \%)$ of the study participants reported a household income between $\$ 20001$ and $\$ 60000$. More than two-thirds (779, $65.9 \%)$ of the study participants were married or living with a partner (Table 1). 
Table 1. Participant characteristics across tertiles of dietary patterns in adults aged 50 years and above, South Australia (Frequency or numbers and percentages; mean values and standard deviations)

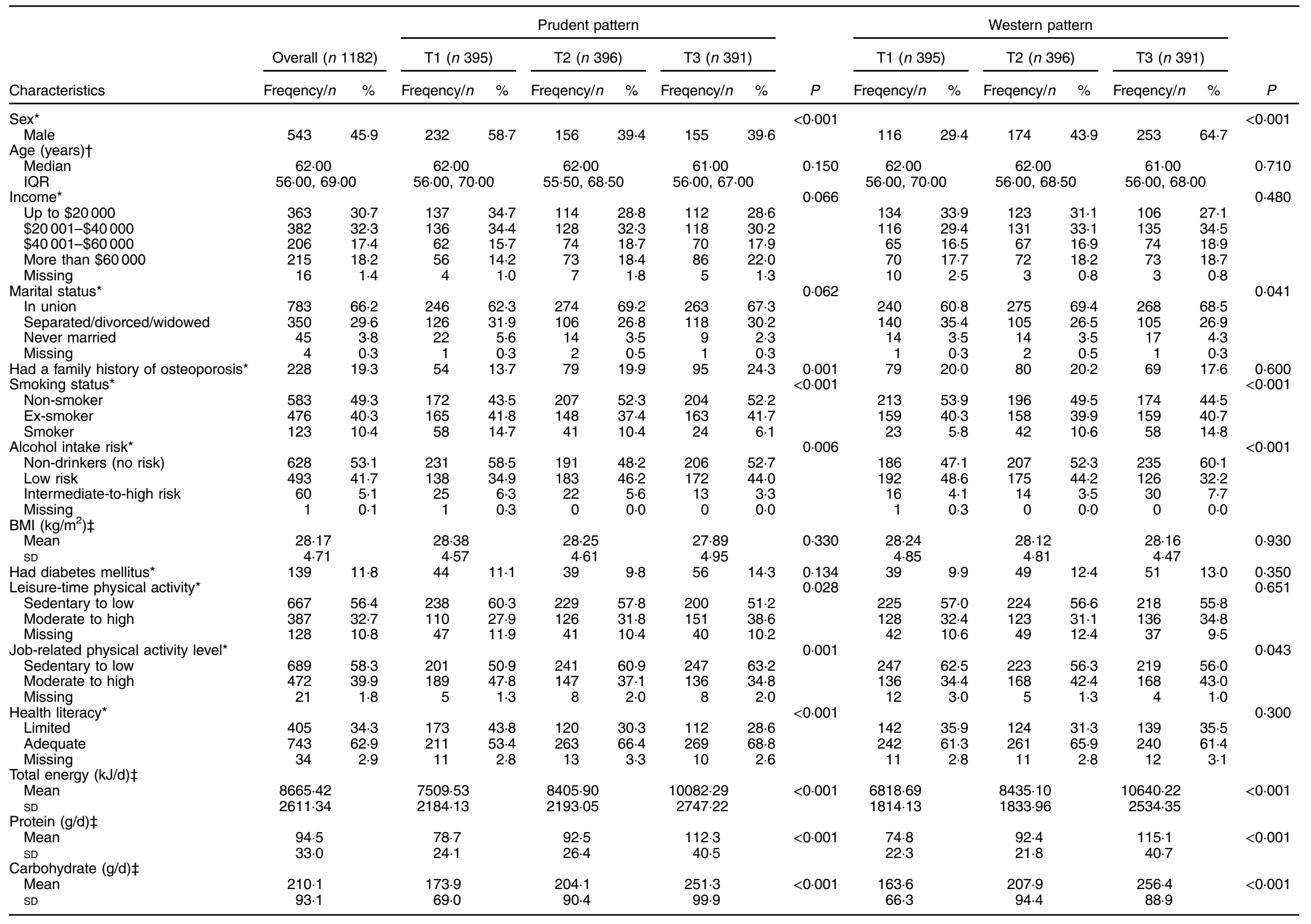




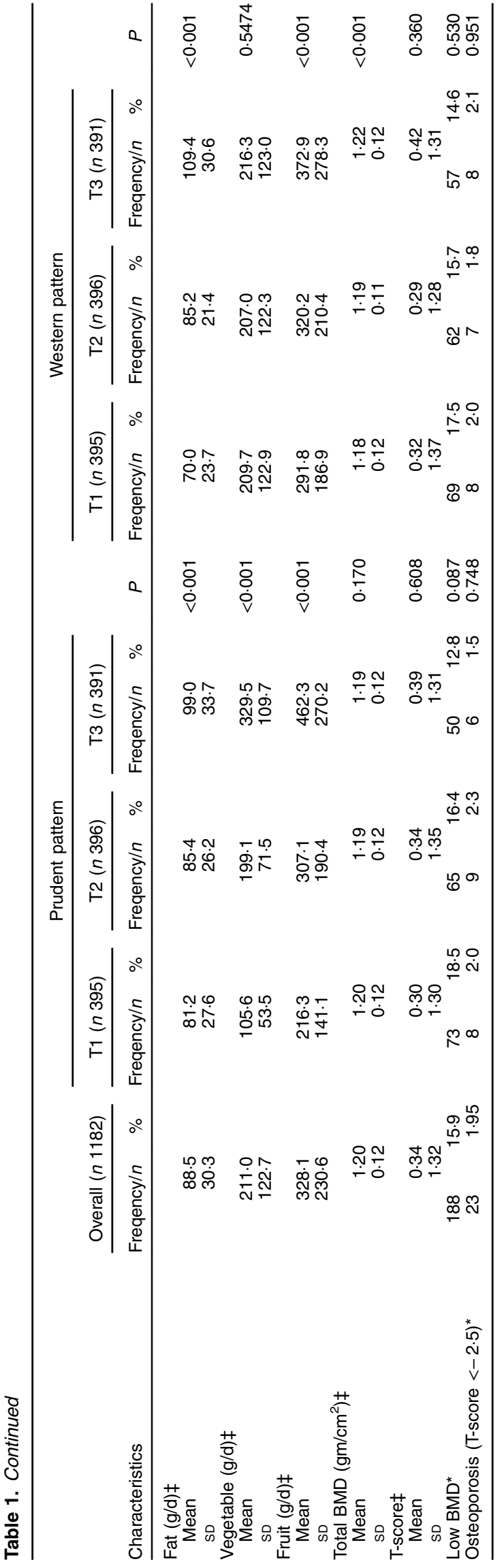

\section{Dietary patterns and characteristics of study participants}

Assessment of dietary misreporting showed that only 7 (0.6\%) participants had under-reporting ${ }^{(2)}$ or over-reporting ${ }^{(5)}$ of EI. We identified two dietary patterns. These patterns explained a total of $17.0 \%$ variance in total food intake $(10.3$ and $6.7 \%$, respectively). Fig. 1 shows the factor loadings for each pattern. Pattern 1 ('prudent pattern') was characterised by high intake of fruits, vegetables, sugar, nut-based milk, fish, legumes and high-fibre bread. In contrast, pattern 2 ('Western pattern') was high in processed and red meat, snacks, takeaway foods, jam, vegemite (a brewers' yeast extract commonly used as a spread in Australia), beer, soft drinks, white bread, poultry, potato with fat, high-fat dairy and eggs. Cross-loading (factor loading $>0 \cdot 30$ in each pattern) was found for sugar, tea and water. Food groups with their constituents are provided in the online Supplementary Table S1.

Socio-demographic characteristics, chronic conditions, EI and BMD across intake levels of the two dietary patterns are shown in Table 1. The overall prevalence of low BMD and osteoporosis was $15.9(12.7 \%$ in men and $18.6 \%$ in women) and $2 \cdot 0 \%$, respectively. More than half $(53.1 \%)$ of the study participants had no risk of harm from alcohol. The mean BMI was $28.2(\mathrm{sD} 4.7) \mathrm{kg} / \mathrm{m}^{2}$. The prevalence of diabetes mellitus was $11 \cdot 8 \%$. The mean whole-body BMD was $1.20(\mathrm{sD} 0 \cdot 12) \mathrm{g} / \mathrm{cm}^{2}$. Family history of osteoporosis was reported in $19 \cdot 3 \%$ of the participants, with almost a quarter $(24.0 \%)$ of study participants in the third tertile (T3) of the prudent pattern having a family history of osteoporosis compared with $13.7 \%$ in the first tertile (T1). More than two-thirds (68.8\%) of participants in T3 of the prudent pattern had adequate health literacy.

There were significant differences in dietary pattern intake by sex, smoking status, alcohol intake risk and job-related PAL. A significant difference in energy, protein, fat, carbohydrate and fruit intakes was found across the tertiles of both dietary patterns. Vegetable intake was significantly different across tertiles of the prudent pattern but not the Western pattern. In addition, family history of osteoporosis $(P<0 \cdot 001)$ and health literacy $(P<0 \cdot 001)$ had crude, significant, positive associations with different levels of the prudent dietary intake. Total BMD $(P<0.001)$ had crude, significant, positive associations with tertiles of the Western dietary pattern.

\section{Dietary patterns and bone mineral density}

The prevalence of low BMD was 18.5, 16.4 and 12.8\% across tertiles of the prudent dietary pattern and $17.5 \%, 15.7$ and $14.6 \%$ across the tertiles of the Western pattern. In the univariate regression analysis, those in $\mathrm{T} 3$ of the prudent pattern had a low prevalence ratio (PR) of low BMD (PR =0.69; $95 \%$ CI 0.48, 0.99) compared with those in T1. There was no crude significant association between Western pattern and low BMD (Table 2).

Significant inverse associations between prudent pattern and low BMD were observed in multivariable regression models (Table 2). After adjustment for socio-demographic and lifestyle factors, chronic conditions and EI, participants in T3 had a significantly lower prevalence of low BMD ( $P R=0.52 ; 95 \%$ CI $0.33,0.83)$ compared with those in T1. No significant 

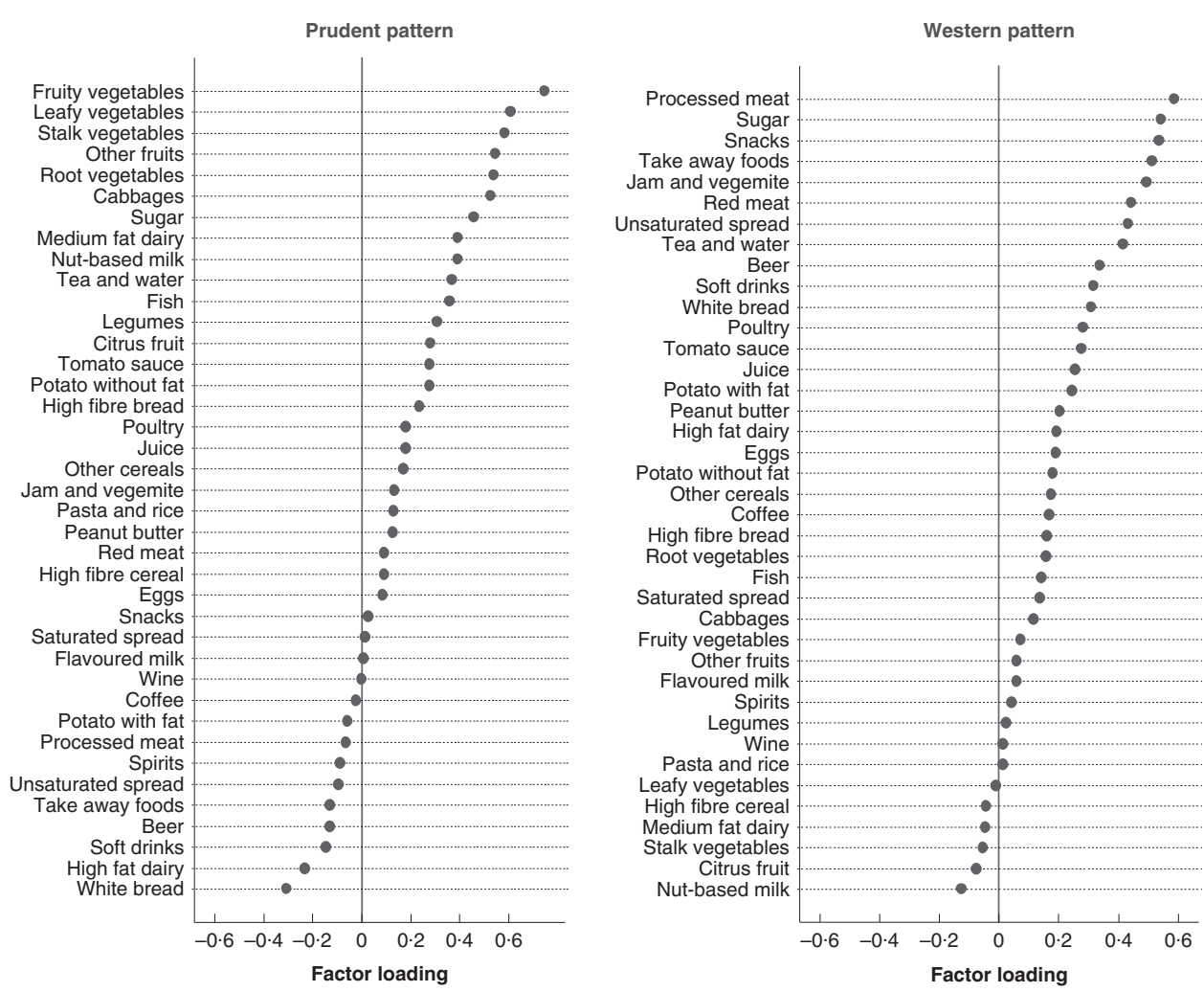

Fig. 1. Factor loadings for two food patterns among adults aged 50 years and above, South Australia ( $n$ 2453).

association between Western pattern and low BMD was observed after adjusting for socio-demographic, lifestyle and chronic condition covariates. However, after adjustment for EI, the study participants in T3 were $68 \%$ more likely to have low BMD ( $\mathrm{PR}=1 \cdot 68$; $95 \%$ CI 1.02, 2.77) compared with those in T1.

We further conducted two sensitivity analyses: (1) by adjusting for season of birth, DXA measurement, vitamin D and Ca supplementation, total number of medications prescribed, sunlight exposure, menopausal status and leisure-time PAL in the final models; and (2) by excluding the missing values of covariates. The association between dietary patterns and low BMD remained in both sensitivity analyses (data not shown).

Interaction was examined between dietary patterns and socio-demographic and lifestyle factors. No interactions were found and these are shown in Fig. 2.

\section{Discussion}

In this study, we identified dietary patterns and the association with low BMD among adults aged 50 years and over. We identified two major dietary patterns: a 'prudent' (healthy) pattern characterised by fruit, vegetables, fish, medium-fat dairy products, nut-based milk, high-fibre bread and legumes and a western pattern characterised by processed and red meat, fast foods (snacks and takeaway foods), soft drinks, white bread and high-fat dairy products. A significant inverse association between prudent pattern and low BMD was observed. In contrast, a positive association between Western pattern and low BMD was found.
The finding that the prudent pattern was inversely associated with low BMD is consistent with previous studies ${ }^{(33,34)}$. The Rotterdam Study in the Netherlands reported that a diet with a high intake of vegetables, fruits, fish, wholegrains, legumes/ beans and dairy products was positively associated with $\mathrm{BMD}^{(33)}$. Among Korean adults, a positive association between a food pattern characterised by high dairy products, fruits and wholegrains and BMD was found ${ }^{(34)}$. It may be that the prudent pattern prevents low BMD because of a large number of food groups within this pattern having high nutrient constituents, such as dairy products ${ }^{(35)}$ and fish $^{(36)}$, and low energy ${ }^{(16)}$, which play an important role in bone mass.

The prudent pattern was also characterised by a high intake of dairy products. A study among postmenopausal women found that high intake of milk and dairy products reduced the risk of osteoporosis ${ }^{(37)}$. Dairy products contain good sources of protein and $\mathrm{Ca}$. Furthermore, the nutrient density of protein, $\mathrm{Ca}$, $\mathrm{Mg}, \mathrm{K}, \mathrm{Zn}$ and $\mathrm{P}$ is higher than any other food. Vitamins, $\mathrm{Ca}$ and polysaccharides are also constituents of nut-based, particularly soyabean, milks ${ }^{(35)}$. In addition, flavonoids in soyabeans, particularly isoflavones, mimic oestrogenic activity and are believed to have an effect in maintaining bone health and in preventing osteoporosis in elderly women ${ }^{(11)}$.

High consumption of fruits and vegetables were also characteristic of the prudent pattern. Fruits and vegetables are comprised of nutrients and non-nutritive substances, such as vitamin $\mathrm{K}, \mathrm{Mg}$, polyphenols and phyto-oestrogens, which are important for bone metabolism ${ }^{(38)}$. Moreover, fruits and vegetables have an alkaline effect due to $\mathrm{Mg}$ andK, which buffers the acidic condition that causes bone resorption ${ }^{(39)}$. 
However, it has been proposed that the effect of fruits and vegetables on bone mass is not due to its buffering nature but rather because of the nutrients (e.g. Ca and vitamin $\mathrm{C}$ ) they contain $^{(40)}$. Current evidence regarding the role of fruits and vegetables in bone health is inconsistent ${ }^{(6,41)}$. The extent of the association between prudent pattern and BMD, due to high intake of fruits and vegetables, requires further investigation and so does the mechanism of action. Nonetheless, public health efforts should target increasing the consumption of vegetables and fruits. In South Australia, the proportion of the population consuming the recommended level of vegetables and/or fruits (consuming $\geq 5$ vegetable servings and/ or $\geq 2$ fruit servings/d) has been consistently approximately $50 \%$ over the past 10 years, among middle-aged and ageing people ${ }^{(42)}$

Although there were no significant interactions between the prudent pattern and the socio-demographic and lifestyle factors in our subgroup analysis, the associations were stronger in certain groups. For instance, in the subgroup of participants who had a family history of osteoporosis, those in T3 of the prudent pattern were found to have significantly lower PR (71\% reduction in PR) of low BMD compared with those in T1. The direction of the association in those who had no family history was also similar, although the magnitude was smaller ( $41 \%$ reduction in PR) and not statistically significant. We also found that the proportion of study participants who had a family history of osteoporosis and adequate health literacy significantly increased across the tertiles of the prudent pattern. Thus, the PR difference between those who had and did not have a family history could be explained by the fact that those who had a family history were aware of their susceptibility to low BMD and reduced the risk by following healthy dietary patterns.

In this study, our analysis showed a significant positive association between Western pattern and low BMD. This was observed after adjustment for all the covariates and EI, showing that the association was independent of EI, which could have arisen from differences in body size, physical activity and metabolic efficiency ${ }^{(43)}$. The association in different subgroups was also consistent with low BMD. Consistent positive associations between similar dietary patterns and low BMD have been reported in previous studies ${ }^{(16,44)}$. Food items (such as soft drinks) in the Western pattern are characterised by low content of important nutrients such as $\mathrm{Ca}$ and high levels of energy content and $\mathrm{P}$, resulting in low serum $\mathrm{Ca}$, which causes bone resorption $^{(45)}$. In addition, evidence shows that high EI is also an important factor in the homoeostasis of nutrients (particularly macrominerals), resulting in reduced $\mathrm{BMD}^{(46)}$.

It is important to recognise some of the important limitations of this study: one of these is the time lapse between collection of dietary and DXA information. Although dietary data were collected between 2008 and 2010, BMD using DXA was determined between 2004 and 2006 with a 4.3-year median difference (minimum $=2 \cdot 8$ and maximum $=6 \cdot 1$ years). Between these years, eating behaviours of the study participants could have changed. Although habits of elderly people in relation to the choice of food groups have been found to be stable over years ${ }^{(47)}$, individuals diagnosed with chronic 


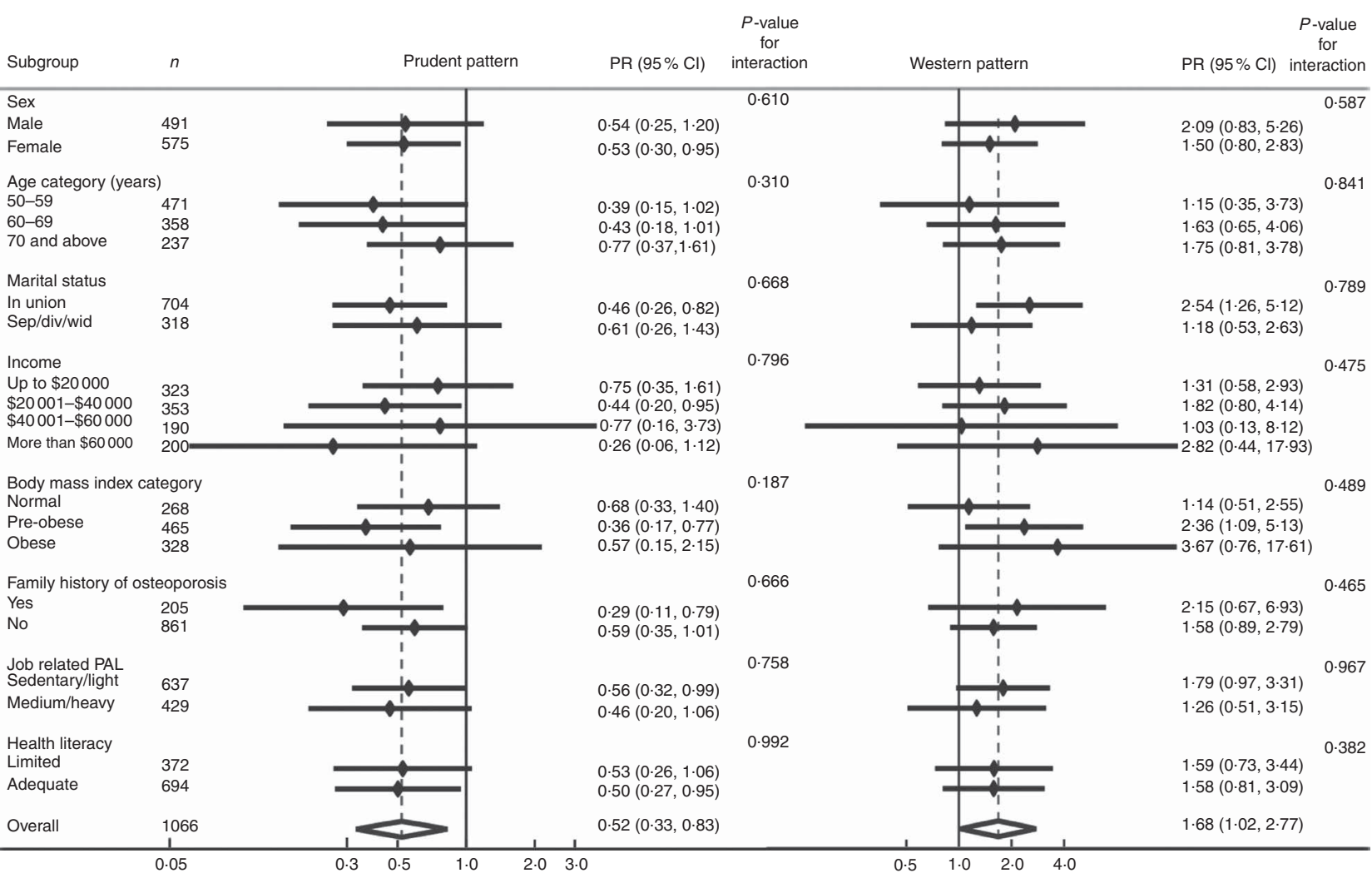

Fig. 2. Subgroup analysis of the association of third tertiles (highest intake) of prudent (left) and Western (right) dietary patterns with low BMD among adults 50 years and above, South Australia. PR, prevalence ratio (adjusted); PAL, physical activity level; sep/div/wid, separated or divorced or widowed. The first tertile (lowest intake of prudent food pattern) was the reference. Poisson's regression was used to compute PR.

diseases may change their diet towards a healthy one, and this may result in an underestimation of estimates. In addition, although studies on the effect of retirement on food habits are limited ${ }^{(48)}$, the available evidence shows that more healthy food habits are likely to be developed among women while it remains similar for men ${ }^{(49)}$. In our study, a total of 175 (14.8\%; $44.9 \%$ men and $55.1 \%$ women) participants retired between the two stages of assessment, which could potentially cause underestimation of the inverse association between prudent dietary pattern and low BMD.

Although FFQ have limitations in providing valid dietary information, they are widely used to measure the usual dietary exposures and behaviours ${ }^{(50)}$. To evaluate the robustness of the dietary data, analysis of dietary misreporting was also conducted to identify misreporting. Furthermore, the dietary analysis we conducted was for a large population group, which can represent the consumption behaviour of the community over time $^{(51)}$. Another potential limitation of this study is the number of cases with missing values of covariates and exclusion of leisure-time PAL from the analysis. However, sensitivity analyses with imputed and excluded covariates suggested that the findings remained similar.

In conclusion, to the best of our knowledge, this is the first study that assessed the association between dietary patterns and BMD among Australians aged 50 years and above. In this community-based study, we found that a dietary pattern characterised by high intakes of fruits and vegetables, medium-fat dairy products and fish was associated with higher BMD. A dietary pattern characterised by high intakes of processed and red meat, fast foods (snacks and takeaway foods), soft drinks, white bread and high-fat dairy products was inversely associated with BMD. Further longitudinal research among ageing populations is warranted.

\section{Acknowledgements}

The authors are thankful to the NWAHS participants for their participation in the study.

The NWAHS was funded by The University of Adelaide, the South Australian Department of Health and The Queen Elizabeth Hospital for which the authors are grateful.

All the authors conceived the study. Y. A. M. conducted all the analyses and wrote all drafts of the paper. Z. S. assisted with analysis and reviewed and provided comment on all drafts. T. K. G. and R. A. reviewed and commented on all drafts. All the authors read and approved the final manuscript.

The authors have no financial or personal conflicts of interest to declare.

\section{Supplementary material}

For supplementary material/s referred to in this article, please visit http://dx.doi.org/doi:10.1017/S0007114516003366 


\section{References}

1. Manolagas SC \& Jilka RL (1995) Bone marrow, cytokines, and bone remodeling. Emerging insights into the pathophysiology of osteoporosis. N Engl J Med 332, 305-311.

2. Cooper C, Campion G \& Melton LJ 3rd (1992) Hip fractures in the elderly: a world-wide projection. Osteoporos Int 2, 285-289.

3. Gill T, Martine T, Laslett L, et al. (2008) An Epidemiological Analysis of Osteoporosis among South Australian Adults. Adelaide: Population Research and Outcome Studies Unit, SA Health.

4. Australian Bureau of Statistics (2012) Australian Health Survey, 2011-2012. Canberra: Australian Bureau of Statistics.

5. Kanis JA (1996) Testbook of Osteoporosis. Oxford: Blackwell Science.

6. Sahni S, Mangano KM, McLean RR, et al. (2015) Dietary approaches for bone health: lessons from the Framingham Osteoporosis Study. Curr Osteoporos Rep 13, 245-255.

7. Wei P, Liu M, Chen Y, et al. (2012) Systematic review of soy isoflavone supplements on osteoporosis in women. Asian Pac J Trop Med 5, 243-248.

8. Caroli A, Poli A, Ricotta D, et al. (2011) Invited review: dairy intake and bone health: a viewpoint from the state of the art. J Dairy Sci 94, 5249-5262.

9. Skowronska-Jozwiak E, Jaworski M, Grzywa A, et al. (2014) Influence of calcium intake on bone mineral density and incidence of fractures in treatment-naive women from Lodz urban area - a part of EPOLOS study. Ann Agric Environ Med 21, 201-204.

10. Mangano KM, Sahni S, Kerstetter JE, et al. (2013) Polyunsaturated fatty acids and their relation with bone and muscle health in adults. Curr Osteoporos Rep 11, 203-212.

11. Messina MJ (1999) Legumes and soybeans: overview of their nutritional profiles and health effects. Am J Clin Nutr $\mathbf{7 0}$, 439s-450s.

12. Newby PK \& Tucker KL (2004) Empirically derived eating patterns using factor or cluster analysis: a review. Nutr Rev $\mathbf{6 2}$, 177-203.

13. Michels KB \& Schulze MB (2005) Can dietary patterns help us detect diet-disease associations? Nutr Res Rev 18, 241-248.

14. Lim YS, Lee SW, Tserendejid Z, et al. (2015) Prevalence of osteoporosis according to nutrient and food group intake levels in Korean postmenopausal women: using the 2010 Korea National Health and Nutrition Examination Survey Data. Nutr Res Pract 9, 539-546.

15. Langsetmo L, Poliquin S, Hanley DA, et al. (2010) Dietary patterns in Canadian men and women ages 25 and older: relationship to demographics, body mass index, and bone mineral density. BMC Musculoskelet Disord 11, 20.

16. McNaughton SA, Wattanapenpaiboon N, Wark JD, et al. (2011) An energy-dense, nutrient-poor dietary pattern is inversely associated with bone health in women. J Nutr 141, $1516-1523$.

17. van den Hooven EH, Ambrosini GL, Huang R-C, et al. (2015) Identification of a dietary pattern prospectively associated with bone mass in Australian young adults. Am J Clin Nutr 102, 1035-1043.

18. Grant JF, Taylor AW, Ruffin RE, et al. (2009) Cohort profile: The North West Adelaide Health Study (NWAHS). Int J Epidemiol 38, 1479-1486.

19. Grande ED \& Taylor AW (2010) Sampling and coverage issues of telephone surveys used for collecting health information in Australia: results from a face-to-face survey from 1999 to 2008. BMC Med Res Methodol 10, 1-11.
20. Giles GG \& Ireland PD (1996) Dietary Questionnaire for Epidemiological Studies (Version 2). Melbourne: The Cancer Council Victoria.

21. Schoenaker DAJM, Dobson AJ, Soedamah-Muthu SS, et al. (2013) Factor analysis is more appropriate to identify overall dietary patterns associated with diabetes when compared with treelet transform analysis. J Nutr 143, 392-398.

22. National Heart Foundation, Australian Institute of Health and Welfare (1989) Risk Factor Prevalence Study: Survey no. 3. Canberra: NHF.

23. World Health Organization (1995) Physical status: the use and interpretation of anthropometry. Report of a WHO Expert Committee, WHO Technical Report Series no. 854. Geneva: WHO.

24. Australian Bureau of Statistics (1995) National nutrition and physical activity survey questionnaire. Canberra: ABS. http:// www.abs.gov.au/AUSSTATS/abs@.nsf/DetailsPage/4363.0.55. 0012011-13? OpenDocument (accessed Janaury 2016).

25. D’Onise R, Shanahan EM, Gill T, et al. (2010) Does leisure time physical activity protect against shoulder pain at work? Occup Med 60, 383-388.

26. Weiss BD, Mays MZ, Martz W, et al. (2005) Quick assessment of literacy in primary care: the newest vital sign. Ann Fam Med 3, 514-522.

27. Baker DW, Williams MV, Parker RM, et al. (1999) Development of a brief test to measure functional health literacy. Patient Edu Coun 38, 33-42.

28. Appleton SL, Seaborn CJ, Visvanathan R, et al. (2013) Diabetes and cardiovascular disease outcomes in the metabolically healthy obese phenotype: a cohort study. Diabetes Care 36, 2388-2394.

29. World Health Organization (1994) Assessment of Fracture Risk and its Application to Screening for Postmenopausal Osteoporosis. Report of a WHO Study Group, World Health Organization Technical Report Series no. 843. Geneva: WHO.

30. Goldberg G, Black A, Jebb S, et al. (1991) Critical evaluation of energy intake data using fundamental principles of energy physiology: 1 . Derivation of cut-off limits to identify underrecording. Eur J Clin Nutr 45, 569-581.

31. Black AE (2000) Critical evaluation of energy intake using the Goldberg cut-off for energy intake: basal metabolic rate. A practical guide to its calculation, use and limitations. Int $J$ Obes 24, 1119-1130.

32. Barros AJ \& Hirakata VN (2003) Alternatives for logistic regression in cross-sectional studies: an empirical comparison of models that directly estimate the prevalence ratio. $B M C$ Med Res Methodol 3, 21.

33. de Jonge EA, Kiefte-de Jong JC, de Groot LC, et al. (2015) Development of a food group-based diet score and its association with bone mineral density in the elderly: the Rotterdam study. Nutrients 7, 6974-6990.

34. Shin S, Sung J \& Joung H (2015) A fruit, milk and whole grain dietary pattern is positively associated with bone mineral density in Korean healthy adults. Eur J Clin Nutri 69, 442-448.

35. Bowes A, Church H \& Pennington J (1994) Bowes and Church's Food Values of Portions Commonly Used. Philadelphia, PA: Lippincott.

36. Chen Y-m, Ho S \& Lam S (2010) Higher sea fish intake is associated with greater bone mass and lower osteoporosis risk in postmenopausal Chinese women. Osteoporos Int 21, 939-946.

37. Shin S \& Joung H (2013) A dairy and fruit dietary pattern is associated with a reduced likelihood of osteoporosis in Korean postmenopausal women. Br J Nutr 110, 1926-1933.

38. Tucker KL (2009) Osteoporosis prevention and nutrition. Curr Osteoporos Rep 7, 111-117.

39. Maurer M, Riesen W, Muser J, et al. (2003) Neutralization of western diet inhibits bone resorption independently of 
$\mathrm{K}$ intake and reduces cortisol secretion in humans. Am J Physiol Renal Physiol 284, F32- F40.

40. Macdonald HM (2007) Influence of organic salts of potassium on bone health: possible mechanisms of action for the role of fruit and vegetables. Int Congress Ser 1297, 268-281.

41. Hamidi M, Boucher BA, Cheung AM, et al. (2011) Fruit and vegetable intake and bone health in women aged 45 years and over: a systematic review. Osteoporos Int 22, $1681-1693$.

42. Taylor AW, Dal Grande E, Wu J, et al. (2014) Ten-year trends in major lifestyle risk factors using an ongoing population surveillance system in Australia. Popul Health Metrics 12, 1.

43. Willett W (2013) Nutritional Epidemiology. New York: OUP.

44. de Franca NA, Camargo MB, Lazaretti-Castro M, et al. (2015) Dietary patterns and bone mineral density in Brazilian postmenopausal women with osteoporosis: a crosssectional study. Eur J Clin Nutr 70, 85-90.

45. Amato D, Maravilla A, Montoya C, et al. (1998) Acute effects of soft drink intake on calcium and phosphate metabolism in immature and adult rats. Rev Invest Clin 50, 185-189.
46. Tucker KL, Morita K, Qiao N, et al. (2006) Colas, but not other carbonated beverages, are associated with low bone mineral density in older women: the Framingham Osteoporosis Study. Am J Clin Nutr 84, 936-942.

47. Jankovic N, Steppel MT, Kampman E, et al. (2014) Stability of dietary patterns assessed with reduced rank regression; the Zutphen Elderly Study. Nutr J 13, 1-9.

48. Zantinge EM, van den Berg M, Smit HA, et al. (2014) Retirement and a healthy lifestyle: opportunity or pitfall? A narrative review of the literature. Eur J Public Health 24, 433-439.

49. Helldan A, Lallukka T, Rahkonen O, et al. (2012) Changes in healthy food habits after transition to old age retirement. Eur J Public Health 22, 582-586.

50. Smith W, Mitchell P, Reay EM, et al. (1998) Validity and reproducibility of a self-administered food frequency questionnaire in older people. Aust N Z J Public Health 22, 456-463.

51. Newby PK, Weismayer C, Åkesson A, et al. (2006) Long-term stability of food patterns identified by use of factor analysis among Swedish women. J Nutr 136, 626-633. 